\title{
A Generic Execution Management Framework for Scientific Applications
}

\author{
Tanvire Elahi, Cameron Kiddle and Rob Simmonds \\ Department of Computer Science \\ University of Calgary \\ Calgary, Alberta, Canada \\ \{telahi,kiddlec,simmonds\}@cpsc.ucalgary.ca
}

\begin{abstract}
Managing the execution of scientific applications in a heterogeneous grid computing environment can be a daunting task, particularly for long running jobs. Increasing fault tolerance by checkpointing and migrating jobs between resources requires expertise and time of the scientist. Automation of such tasks can allow the scientist to focus more on the scientific results and less on the technical details.

In this paper a generic framework for managing and automating the execution of jobs is presented. It uses of a variety of information models describing systems, policies, and application details/requirements to make suitable decisions on where and how to run, checkpoint, migrate and reconfigure jobs as needed. To demonstrate the utility of the framework, it is used as part of a simulation study to assess the impact availability of application memory usage information has on meeting the QoS objectives of job submitters and on overall utilization of resources. The study shows that with greater availability of memory usage information, the execution management framework is able to better meet user objectives and improve utilization of resources, particularly when the objective is to make more efficient use of resources.
\end{abstract}

Keywords: Application Modelling, Grid Computing, Automation, Execution Management, Simulation

\section{Introduction}

Grid computing technologies enable the sharing of computing resources and greater collaboration among research communities. Increased availability of high performance resources has paved the way for researchers to conduct sophisticated computational and resource intensive experiments. Many of these experiments involve the execution of computational jobs that often run for long periods of time. Management of the execution of these jobs has proven to be a complex issue due to the dynamics and heterogeneity of the environment. Often, jobs need to be checkpointed, migrated and reconfigured to improve the fault tolerance and to satisfy scheduling constraints on different resources.

Due to the complexity, execution of long running jobs in grid environments continues to require a high level of expertise. Often, active intervention from the user's part is required to perform the necessary steps of execution management. Not only does a user need to be vigilant enough to adapt to the dynamics of the environment, he is also expected to have knowledge of the application's capabilities and resource usage behaviours. Mechanisms to automate execution management are necessary to allow researchers to focus on experiment results rather than the underlying mechanics of executing the application. In this work, we propose a generic framework that supports unattended, reliable, and efficient execution of resource intensive scientific applications.

Previously, we developed a meta-scheduling service [2] capable of dynamically reconfiguring jobs to best suit the resources they are submitted to. It addresses some aspects of automation by using common information models, defined by the GRC Model Schema [7, 8], that describe systems, scheduling policy and basic information on installed applications. However, the models do not provide details on how the applications can be reconfigured or on what the resource requirements of the applications are. To address the shortcomings, we proposed a model in [3] that describes an application's reconfigurability on various execution scenarios and the processes that need to be carried out in order for the application to use a new configuration. The model was extended in [4] to include a memory requirement specification that describes an application instance's memory usage behaviour on different configurations. However, an integrated system incorporating the model components was not materialized. 
This paper presents a prototype implementation of a generic framework that supports execution management of jobs on heterogeneous resources. The framework is an extension to the one discussed in [2] in multiple aspects. Firstly, the functionalities of the modules are driven by the application models describing the configurability and memory usage pattern, in addition to the system description captured by the GRC Model Schema. Use of models abstracts the heterogeneity of both the resources and the applications and makes the framework functional without the need to be tailored to any specific application, as was done in [2]. Also, in [2], the focus was on the ability of the framework to dynamically reconfigure jobs, not the intelligent selection of configurations. The proposed framework is equipped with an adaptive configuration selection service which is guided by Quality of Service (QoS) requirements of the job submitter.

The prototype system has been used to study how information about the memory usage behaviour of jobs can facilitate the process of discovering and selecting configurations at different phases of a job's lifecycle. Particularly, we study the impact memory usage information has on meeting the job submitters QoS objectives and on the overall utilization of the resources.

The paper is organized as follows. Section 2 discusses some existing execution management and modelling approaches. Section 3 illustrates the architecture of the proposed execution management system and how different parts of the models play a role. Section 4 discusses the experimental results we obtained simulating various job execution scenarios. Conclusions and future work are discussed in Section 5.

\section{Related Work}

We focus on existing works aimed at execution management of scientific jobs and how the systems deal with the application's resource requirements. GrADS $[1,12]$ and GridWay [6] are examples of frameworks for automating the execution of jobs. Both of these frameworks provide support for dynamic reconfiguration of applications to different extents. However, they are aimed at the development of applications that make use of particular checkpointing, migration and reconfiguration mechanisms that are part of the framework and thus have not focused much on the modelling of applications. We aim to automate the execution of existing unmodified applications that may have various mechanisms to support checkpointing, migration and reconfiguration. As such, models for describing these mechanisms are crucial to developing a general solution.

GRMS (Grid Resource Management and Brokering Service) [9] is another implementation of an adaptive execution management system, developed as a part of GridLab [11], a suite for higher-level services for grid. Although it does not explicitly support reconfiguration of jobs, it provides mechanisms to dynamically reschedule jobs by using application-level checkpointing. As is the case for GrADS and GridWay, applications must be modified or built to use the checkpointing functionality specific to GRMS.

A model based deployment framework was proposed in [10]. Programs written in a particular programming model (MPI, GridCCM) are converted and deployed on different grid nodes based on the architectural requirements of the individual parts. However, no notion of application reconfiguration is discussed as the configuration parameters (i.e., number of processors) are included in the description for each of the entities.

Most of the aforementioned works either do not explicitly incorporate a job's memory requirement behaviour within the framework or hard code the requirement as a static parameter into the job specification. GridWay provides mechanisms for dynamically updating the memory requirement expression and uses it for resource selection. However, the application needs to be customized first so that it is able to perform this functionality where our approach is designed to work with an unmodified application. Also, the memory specification used by GridWay is very simple, capturing only the total memory requirements of the job with no scalability information for parallel jobs using different numbers of processors. The proposed framework in this paper deals with a more comprehensive specification of memory usage pattern.

\section{System Architecture \& Functionalities}

This section discusses the proposed Execution Management System. We begin with the architecture of the system and an overview of different modules and their functionalities. Following that, we briefly discuss different model components used by the modules. A detailed discussion on the configuration selection functionality of the framework is also provided. 


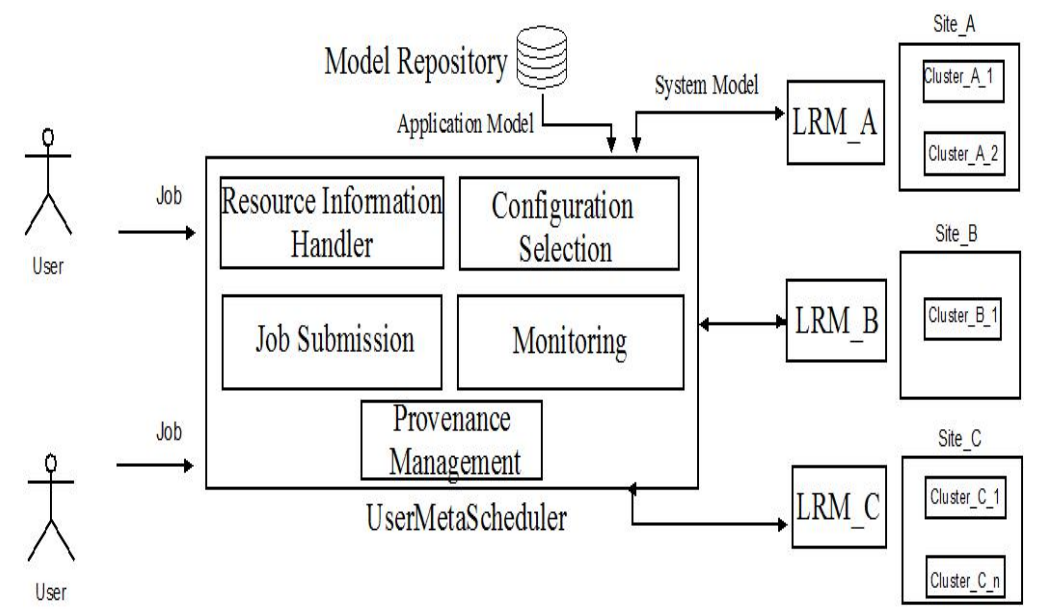

Figure 1. EMS Architecture

\subsection{Architecture}

Figure 1 shows the overall architecture of the proposed system. It also shows the interaction between different components of the framework. We provide brief descriptions of the components and their functionalities. We begin with the discussion on the Local Resource Manager (LRM) Adaptor followed by a detailed description of the UserMetaScheduler module.

\subsubsection{LRM Adaptor}

The LRM Adaptor is responsible for communicating with the Local Resource Manager (LRM) managing the resources. The functionalities typically carried out by the LRM Adaptor are:

- Providing static information about the resource's physical configuration and usage policies to the UserMetaScheduler module.

- Estimate the QWT based on the resource requirements of the job.

- Communicate with the monitor of the UserMetaScheduler about status of the submitted jobs. Also it extracts and provides information about the resource usage of a completed job.

\subsubsection{UserMetaScheduer}

This module works as a gateway for jobs submitted by the user. It is responsible for managing the overall execution of the jobs on behalf of the user. Functionalities provided by this module include:

- Information handling: Involves obtaining publicly available information about potential resources. The information is described using a schema. The handler is responsible for parsing and interpreting the information.

- Configuration discovery \& selection: Using a resource's physical description and policy constraints, and the application configurability, the module discovers possible ways of executing the job and makes a selection. The configuration selection mechanism is discussed in detail in Section 3.3.

- Job submission: In addition to submitting jobs to an appropriate resource, it is also determined if any preparation steps are required to enable the job to use the selected configuration. For example, sometimes a job's input file needs to be transformed to resume the job on a different number of processors.

- Monitoring: The module obtains information through the LRM Adaptor about the status of the submitted jobs. It also extracts provenance information about them.

- Management of provenance information: Data of particular interest includes the configuration used by the job, the submission time, start time and resource usage. 


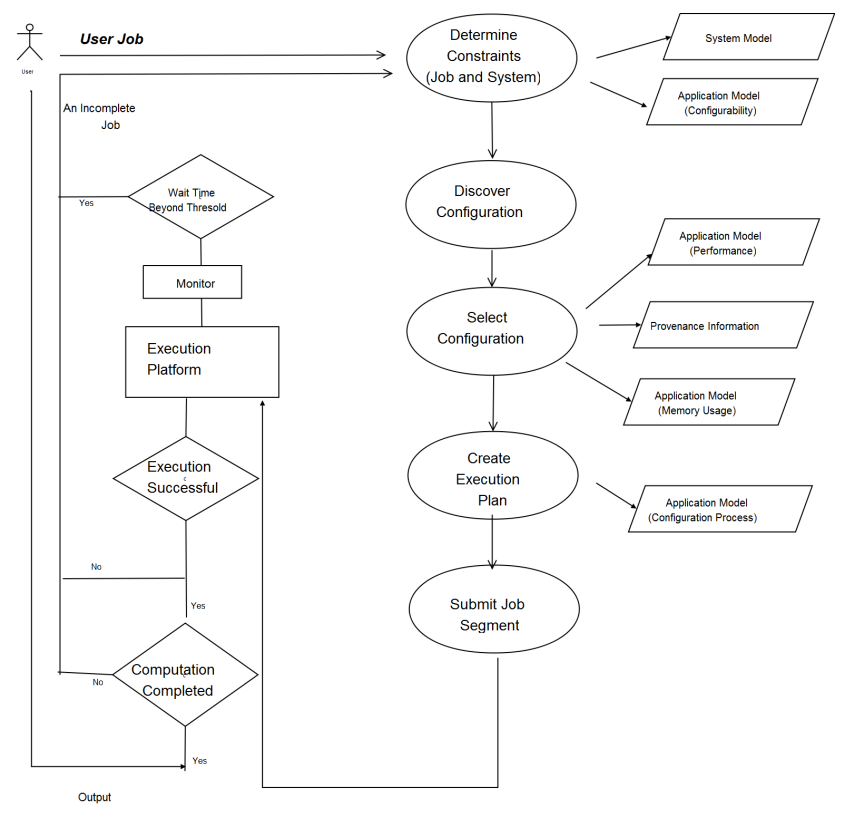

Figure 2. Job lifecycle

The framework uses an approach to manage the execution of long running jobs that was initially proposed in [2]. In this approach, a long job is split into multiple shorter segments by performing periodic checkpointing and resubmission. We observe a twofold benefit of using this approach. Firstly, it allows a job to adhere to the walltime constraints of the resources. Secondly, it makes the execution management processes more adaptive to the resource dynamics and adds more fault tolerance. As a grid environment is prone to system errors and failures, running shorter jobs results in a smaller loss of computation in case of such failures.

Figure 2 captures the phases a job goes through during it's lifecycle. It also shows model information that is required at various points in the lifecycle. Models are used in the configuration discovery process to find different ways a job can be executed at a particular phase of it's lifecycle. The memory requirement specification tells how the job will use the memory resources. The information is important in configuration discovery selection as it helps in finding the resources that satisfy the memory requirement of the job. It also allows a resource usage efficiency analysis to be conducted for the configurations. The following subsection provides a brief overview of the models used by the framework for managing the execution of jobs.

\subsection{Models}

The functionality of the framework is driven by the models describing resources and different aspects of the applications used by the jobs. The following model components are of particular interest to the framework.

\subsubsection{System Model}

The GRC Schema [7] provides a common information model to describe the resources, their physical configuration and usage policies. Use of the model enables interoperation and also facilitates use of tools transparent to the underlying heterogeneity of the resources.

\subsubsection{Application Model}

The application model [3] is designed to describe various deployment related aspects of the applications. Included among the descriptions are the constraints on the execution of the application, reconfigurability at different phases of execution which essentially indicates the checkpointing and migration capability of the jobs using the application. Often, using a new configuration for a job requires some preparation steps to be carried out. Such steps are captured by the application model. 


\subsubsection{Memory Usage Model}

The objective of this model is to help predict the memory usage behaviour of the jobs using a particular application. The existing model [4] captures four major aspects of the memory usage behaviour, i) how various job parameters affect the amount of memory used by the job when executing sequentially, ii) how different processes differ in terms of the amount of memory used, iii) how the memory usage scales with an increasing number of processors used by the job and iv) profile of memory usage, i.e., the changes in the amount of memory used at different phases of a job's execution.

From our experience with different scientific applications, they differ in levels on which certain aspects of memory usage pattern is understood. The framework is designed in a way so that it can adapt based on the amount of information available. Also it is able to incrementally fill in missing pieces of the model using the usage details of the initial iterations of jobs.

\subsection{Configuration Selection}

One of the most important functionalities provided by the UserMetaScheduler module is selecting a single configuration from a list of options for executing a job. As the problem of configuration selection is aimed at satisfying both user and resource oriented objectives it is often difficult to find an optimum solution. In this work, we have used a set of heuristics to do that. The following factors are considered important in order for the heuristics to function:

- Optimization objective of the job submitter: Currently, we are considering two simple but commonly used optimization objectives. The first one attempts to minimize the overall completion time of the job. The jobs using this objective are classified as Urgent jobs. The second type of optimization is of interest for the user who is less concerned about the completion time and more interested in maximizing the usage of the acquired resource (both processors and memory). Intrinsically, jobs of this category aim at finding a balance between the cost and the overall completion time. They are classified as Economic jobs.

- Performance of the application: When selecting a resource, it is important to predict the performance the job will exhibit on different resources and using different numbers of processors ( $n p)$. We have used a simple application performance model where the performance is described by two parameters, i) sequential performance of the application in comparison to any reference architecture and ii) the scalability of the application for different $n p s$ on different resources. Also, for the time being, we assume the performance as a monolithic function having a similar scaling pattern over the job's lifetime. However, the framework is able to deal with a more sophisticated performance description.

- Probable start time: The estimation on the Queue Wait Time (QWT) is important to minimize the possibility of starvation for jobs. The estimation is done by the LRM considering the jobs that are running on the system and also jobs waiting in the queue.

- Resource requirements: For this work, we have particularly focused on a job's memory requirements. Knowledge about a job's memory usage enables estimation of the costs of executing the job using different configurations. It also helps perform a utilization analysis for the available configuration options.

- Cost: It represents the cost a user incurs for using resources for a duration of time. The price for a unit of resource time can be defined in terms of money or any other commodity.

\subsubsection{Selection Process}

The selection process is primarily guided by the optimization objective chosen by the user. Though the process expects some key information about the application, it is able to deal with different information availability scenarios.

A configuration is comprised of the following attributes, i) name of the host, ii) name of the cluster, iii) attributes describing the architecture of the node, iv) number of processors (np) to be used, v) the amount of memory that can be requested with each processors and vi) walltime, i.e., the time the job will run using the configuration. The process of selecting a single configuration from all the possible configurations is carried out in three steps. Initially, the configurations are filtered such that only the configurations that can meet the memory requirement are selected. The shortlisted configurations are then ranked according to the user's optimization objective. Following that, configuration with the highest rank value is chosen. A detailed description of these steps is provided: 


\begin{tabular}{|c|c|c|c|}
\hline QoS & Level1 & Level2 & Level3 \\
\hline Economic & Efficiency $(\uparrow)$ & QWT $(\downarrow)$ & np $(\uparrow)$ \\
\hline Urgent & work_done $(\uparrow)$ & efficiency $(\uparrow)$ & QWT $(\downarrow)$ \\
\hline
\end{tabular}

Table 1. Ranking Hierrarchies

\section{Filtering}

Whether or not to include a configuration in the shortlist is determined by checking if the nodes have enough memory to accomodate the job should the configuration be used, i.e., the size of the node is larger than the per processor memory usage of the job on that configuration.

The process is straightward in the case where memory usage information is available. In absence of the memory

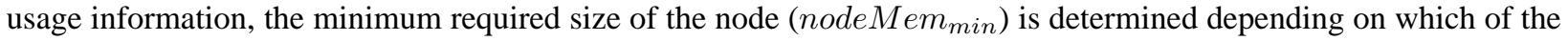
following three situations applies to the job:

- First iteration, first attempt: If this is the first iteration of the job and the job is being submitted for the first time, no minimum size is specified.

- First iteration, previous failures: If the 'np' of the configuration is smaller than or equal to any of the 'np's on which the job was attempted before but was unsuccessful, the value of nodeMem min $_{\text {in }}$ is calculated the following way:

$$
\text { nodeMem }_{\text {min }}=2 \times \max \left(m p p_{1}, m p p_{2}, \ldots, m p p_{n}\right)
$$

Where $m p p_{i}$ represents the amount of memory requested on an unsuccessful attempt.

- Subsequent iterations: If the 'np' of the configuration is greater than or equal to any of the 'np's that was used successfully by the job, the value of the node $\mathrm{Mem}_{\min }$ is calculated the following way:

$$
\text { nodeMem }_{\text {min }}=\min \left(m p p_{1}, m p p_{2}, \ldots, m p p_{n}\right)
$$

Where $m p p_{i}$ represents the amount of memory used on a successful attempt. In case of the presence of the profile information, the value is adjusted to represent the amount of memory the job would use if it were in the current memory usage phase.

In all other cases, no minimum value for node $\mathrm{Mem}_{\min }$ is specified.

The above-mentioned filtering techniques cover most of the cases of non-availability of information, except for the cases when sequential memory usage is available for Urgent jobs. A conservative approach is taken in calculating the nodeMem $_{\text {min }}$ for such jobs. The value of the node $\mathrm{Mem}_{\min }$ is determined by an iterative process where initially it is set to the amount of memory the job would use sequentially. In case of unavailability of nodes with such a high amount of memory, the value is reduced to half of it's previous value. The process is continued until nodes are found that meet the criteria.

\section{Ranking}

We have used a hierarchical scale for ranking the configurations in which a factor is only considered when two configurations have equal values for all the factors from levels above it.

Table 1 gives an overview of the ranking scheme. The arrows indicate whether to maximize/minimize the factor. For Urgent jobs, the primary consideration is given to the amount of computation that is expected to be completed using a configuration (work_done). The value of this factor is computed the following way:

$$
\frac{\text { walltime } \times \text { speedup }}{\text { walltime }+Q W T_{n}}
$$

where speedup $_{n}$ represents the ratio of sequential and parallel runtime using 'n' processors and $Q W T_{n}$ is the approximated wait time for 'n' processors on a given system.

For Economic jobs, the objective of the user is to maximize the usage of the resources to be acquired while maintaining an acceptable execution speed. The major parameter considered in ranking is the overall usage efficiency of CPU and memory of a configuration. The metric is calcualted the following way:

$$
\left(w_{m e m} \times m e m_{e f f}+w_{p r o c} \times \operatorname{proc}_{e f f}\right)
$$


where $m e m_{e f f}$ and $\operatorname{proc}_{\text {eff }}$ indicate the memory and processor usage efficiency of the configuration, respectively, and $w_{m e m}$ and $w_{\text {proc }}$ represent the weights to be given on memory and processor usage. The $\operatorname{proc}_{e f f}$ and $m e m_{e f f}$ are computed as follows:

$$
\text { proc }_{e f f}=\frac{\text { speedup }_{n p}}{n p \times P E}
$$

where $n p$ is the number of processors to be used for the configuration, speedup ${ }_{n p}$ represents the ratio of sequential and parallel runtime using 'np' processors and Processor Equivalency (PE) is computed the following way:

$$
P E=\left\lceil\frac{\text { mpp }(\text { Requested })}{\text { default_allocation }}\right\rceil
$$

Where the defaultallocation is the amount of memory allocated for each of the processors on a given resource. Typically, it is the ratio of the amount of memory on each of the nodes and the number of processors within it.

$$
\text { mem }_{\text {eff }}=\frac{m p p(U s e d)}{\max (m p p(\text { Requested }), P E \times \text { default_allocation })}
$$

Similar weight is given to processor and memory usage in the presence of both performance and memory usage information. In absence of the memory usage information, full weight is given on the processor efficiency.

\subsubsection{Refinement}

Once selected, the configuration may need to go through some refinement processes. Two parameters from the selected configuration are of interest, i) the amount of memory to be requested and ii) the walltime.

- Memory: If the memory usage amount is known for the selected configuration, the parameter is adjusted accordingly. For other cases, this parameter is modified only for jobs that has completed one or more iterations successfully with the assumption that, per processor memory requirement goes downwards with an increasing number of processors, if the 'np' of the chosen configuration is greater than or equal to any of the 'np's previously used, the amount of memory used on that configuration is requested.

- Walltime: Whether or not to modify this parameter, depends on the optimization objective of the user and the availability of information on memory requirements. Often, the amount of memory used by a job changes during it's lifetime. As mentioned, such behaviour is captured in the memory model [4]. In the presence of this profile information, the module responsible for configuration selection attempts to further optimize the memory usage efficiency by executing the job segment long enough to finish the current phase of the profile. So, in cases where the default walltime of the configuration overruns the phase, the length of the segment is adjusted (curtailed). In most cases, such refinement is not performed for Urgent jobs with an anticipation that it will incur more delay due to frequent preemption and resubmission.

\subsubsection{Dynamic Learning of Memory Usage Pattern}

The UserMetaScheduler is able to adapt to non-availability or partial availability of information on memory requirements of jobs. As discussed in the previous section, in case of non availability, a trial-and-error strategy is used to determine the amount of memory to be requested. In cases where partial information is available, the system attempts to use the initial iterations to dynamically learn about the missing components of the memory usage behaviour and use it for the subsequent iterations. However, the way the job passes through the learning phase would depend on the job's optimization objective. For instance, for jobs with information on scaling behaviour, in absence of information on sequential usage, Economic jobs use the smallest possible number of processors for the first iteration to find out the sequential memory usage information. Such a cost saving approach is not used by the Urgent jobs as they aim to minimize the completion time. For these jobs, the configuration selection mechanism used during the initial learning phase does not differ from that used in later phases.

\section{Experiments}

To demonstrate the utility of the proposed execution management framework a prototype implementation has been built and used in a simulation study to assess the impact memory usage information has in the configuration selection process. The experimental methodology used for the simulations is described first, followed by the experimental results and the summary of the observations. 


\begin{tabular}{|c|c|l|l|l|l|l|}
\hline Site name & Cluster name & $\begin{array}{l}\text { Number of } \\
\text { Nodes }\end{array}$ & $\begin{array}{l}\text { Processors per } \\
\text { Node }\end{array}$ & $\begin{array}{l}\text { Memory per } \\
\text { Node (GB) }\end{array}$ & $\begin{array}{l}\text { Cost/Proc. Sec- } \\
\text { ond }\end{array}$ \\
\hline lattice & wg4-wg99 & 96 & 4 & 4 & 1 \\
\hline matrix & m1-m256 & 256 & 2 & 2 & 1 \\
\hline \multirow{2}{*}{ glacier } & ice1_1-ice54_14 & 756 & 2 & 2 & 1 \\
& ice55_1-ice60_14 & 168 & 2 & 4 & 2 \\
\hline robson & r1-r54 & 54 & 2 & 4 & 2 \\
\hline
\end{tabular}

Table 2. Resource Configuration of the Grid

\subsection{Experimental Methodology}

This section describes the methodology used to carry out the experiments. Included are descriptions of the experiment environment, model of the resources and workload, experimental design and the performance metrics.

\subsubsection{Experimental Environment}

The simulation environment is built on SimKit [5], a discrete event simulation kernel developed at the University of Calgary. SimKit uses a logical Process (LP) modelling view of discrete event simulation. In this model, a physical system can be viewed as a set of independent, interacting, concurrently operating components. For the purpose of the experiments, 3 types of LPs have been used. The JobDispatcher LP is responsible for generating the workload and dispatching individual jobs to the UserMetaScheduler LP which implements the functionalities of the module of the framework. The third LP type carries out the functionalities of a Local Resource Manager (LRM). A single instance of each of the JobDispatcher and UserMetaScheduler LPs are used. Separate instances of LRM are used for each of the sites within the modelled grid environment.

\subsubsection{Resource Model}

We modelled a number of resources with different configurations, policy restrictions, and capabilities. Table 2 provides an overview of the physical configurations of the resources modelled for the experiments. Both resource and policy information are based on computer systems from WestGrid (www.westgrid.ca), a high performance computing consortium in Western Canada. Resources differ in terms of the number of processors on a node and the amount of memory they contain. Also, resources imposes different level of constraints on the usage of resources, both in terms of the number of processors given to a job and also the amount of time the resource can be consumed. It is assumed that the scheduling of jobs at the resource level is performed using a 'First-fit, first-served (FFFS)' strategy from a FIFO list, where the first job that will be able to run using the available resources will be started. Also, a simple 'First-fit' processor allocation strategy is used where jobs are given as many processors as possible from a single node.

For the purposes of the simulation study, a simplistic cost model that considers only the ratio of memory per node to processors per node is used. For example, a single processor from the ice1_1-ice54_14 node class (where each node has $2 \mathrm{~GB}$ of memory and 2 processors) will cost 1 unit/second whereas a single processor from the ice55_1-ice60_14 node class (where each node has 4GB of memory and 2 processors) will cost 2 units/second. As much as we recognize the importance of other factors such as processor architecture and interconnect performance in determining the cost of a resource, these would have had little effect on the results as a job's sequential and scaling performance were assumed to be uniform across resources.

\subsubsection{Workload Description}

This section provides an overview of the jobs submitted for execution from the JobDispatcher module. Jobs used for the simulation experiments are characterized in terms of the following parameters:

- Size of the jobs in terms of their memory requirements.

- Information availability scenario on the memory usage.

- Optimization objective (i.e., QoS requirement).

- Run length of the jobs. 


\begin{tabular}{|c|c|c|c|}
\hline QoS & JobSize & Info Availability Type & Walltime (Hours) \\
\hline \multirow{3}{*}{ Economic } & Very Small (0.5GB-1GB) & FullMemInfo & \\
& Small (1GB-4GB) & NoSeq & \multirow{2}{*}{$72-144$} \\
& Medium (4GB-8GB) & NoScaling & \\
\multirow{2}{*}{ Urgent } & Large (8GB-11GB) & OnlyProfile & \\
& Very large (11GB-14GB) & NoInfo & \\
\hline
\end{tabular}

Table 3. Workload Parameters

\begin{tabular}{|c|c|c|c|}
\hline Availability Type & \multicolumn{3}{|c|}{ Information Available } \\
& Sequential & Scaling & Profile \\
\hline FullMemInfo & Yes & Yes & Yes \\
\hline NoSeq & No & Yes & Yes \\
\hline NoScaling & Yes & No & Yes \\
\hline OnlyProfile & No & No & Yes \\
\hline NoInfo & No & No & No \\
\hline
\end{tabular}

Table 4. Memory Usage Information Availability

Table 3 shows the list of possible values for each of the workload parameters. Following is the description of the parameter values:

\section{Size of the job}

This parameter indicates the size of the job in terms of it's memory usage if executed on a single processor. Five categories are used for the experiments. Table 3 shows the range of values for this parameter for each of the categories. A workload of a specific size (e.g., small) contains predominantly jobs of that size (ranges are shown in Table 3), with a smaller fraction of jobs from other size categories. Approximately, $60 \%$ of the jobs come from the specified size category, and $10 \%$ from each of the other size ranges.

\section{Information Availability}

Different information availability scenarios about job's memory usage behaviours are explored. They are shown in Table 4.

\section{QoS Requirements}

The parameter indicates the optimization strategy the job submitter wants the metascheduler to use. As mentioned in the earlier section, we have considered two types of optimization objectives.

\section{Run length}

The length of a job describes the time it will take to complete if the job is executed serially using a reference processor. For all the experiments described in this section, the value of this parameter is chosen within the range given in Table 3.

Workloads used in different experiments are characterized by the first three parameters describes above. The value for the run length parameter follows a uniform distribution within the given range.

For individual jobs within a workload, the value for the 'job size' also follows a uniform distribution within the given size range. In an attempt to minimize the differences between workloads within a given experiment, individual values for these two parameters are kept identical.

An exponential distribution is used to generate the inter-arrival times between the jobs. The mean value used for the inter-arrival time is 200 seconds. The simulations ran for 200000 simulation seconds ( $~ 56$ hours). Like other parameters, same values were used across different workloads.

A single experiment consists of multiple simulations each using a different workload having different combinations of values for the 'job size', 'information availability' and 'QoS requirement' parameter mentioned in the last paragraph. Hence, 50 simulations were carried out for an experiment in order to explore all the scenarios with different job sizes and the information availability. Experiments are run multiple times using different random number seeds. The metrics reported in the later subsections represent the average obtained from all of the runs. Many aspects of a job's behaviour are determined by the characteristics of the corresponding application. Characteristics that we are particularly interested in are the sequential and parallel performance on different systems and memory usage pattern. For simplicity, it is assumed that applications exhibit similar performance. Their memory usage pattern is, however, different. The pattern of memory usage on different number processors in described by 'MemScaleFactor', a number between 0.5 to 0.85 . The higher the value of the factor, the closer the job's memory requirement being linear. For 


\begin{tabular}{|c|c|c|c|}
\hline \multicolumn{2}{|c|}{ Scaling } & \multicolumn{2}{|c|}{ Memory Usage Profile } \\
\hline np & Speedup & PhaseLength (\% of full job length) & $\%$ of Maximum Usage \\
\hline 2 & 2.0 & 10 (Initialize) & 100 \\
4 & 4.0 & 40 (ProcessPhase1) & 70 \\
8 & 8.0 & 50 (ProcessPhase2) & 90 \\
16 & 13.8 & \\
\hline
\end{tabular}

Table 5. Performance and Memory Usage Behaviour of Applications

instance, for a job using 'n' processors, the memory required by each of the processes is calculated the following way:

$$
m p p=\frac{\frac{s e q_{m e m}}{n}}{\text { MemScaleFactor }}
$$

Where $s e q_{m e m}$ is the amount of memory the job would use for sequential execution. The high end of the range for 'MemScaleFactor' was chosen to be 0.85 instead of 1 assuming that, even when executed in parallel, a minimum fraction of the application data to be replicated in all the participating processors. Like some other parameters, the values for the 'MemScaleFactor' follows a uniform distribution within the given range. The same sets of values for these parameters were used across workloads on a given experiment.

Table 5 shows the performance and the memory usage profile of the application used. For different workloads, we have created different information availability scenarios on the memory usage behaviours (Table 4) by making certain parts of that specification unavailable.

\subsection{Metrics}

We have used several key metrics in our simulation to quantify the effect of the parameters on various user and resource objectives. Since individual users and resource owners often have different and possibly conflicting demands, no single measure can comprehensively capture overall grid performance.

\subsubsection{User Level Metrics}

From the users' perspective, key measures of grid performance include the Computation Completed, Cost Incurred and Resource Usage Efficiency. These are computed as follows:

$$
C C=\sum_{k=1}^{n} \text { walltime }_{k} \times \text { speedup }_{n p_{k}}
$$

where CC (Computation Completed) is a measure of the amount of computation completed within a specified time window, $n$ is the number of job segments successfully completed/will complete, walltime indicates the length of the job (in seconds) and speedup ${ }_{n p}$ represents the ratio of the sequential runtime and runtime on 'np' processors.

$$
C I=\sum_{k=1}^{n} \text { walltime }_{k} \times \text { unitCost }_{k} \times P E_{k} \times n p_{k}
$$

Where CI (Cost Incurred) refers to the price users has paid cumulatively within a given time. The unitCost represents the cost/second for each of the Processor Equivalence (PE) used (listed in Table 2) and $n p$ is the number of processors used by the segment.

The following metrics are used to indicate how efficiently the jobs made use of the CPU and memory:

$$
\begin{gathered}
\text { CPUEff }=\frac{\sum_{k=1}^{n} \text { wallime }_{k} * \text { proc }_{\text {eff }}}{\sum_{k=1}^{n} \text { walltime }_{k}} \\
\text { MemEff }=\frac{\sum_{k=1}^{n} \text { walltime }_{k} \times \text { mem }_{\text {ef } f_{k}}}{\sum_{k=1}^{n} \text { walltime }_{k}}
\end{gathered}
$$

where $\mathrm{n}$ is the number of job segments completed or will eventually complete. $\operatorname{proc}_{e f f}$ and $\operatorname{mem}_{\text {eff }}$ are described in Section 3.3.1. 


\subsubsection{Resource Level Metrics}

A resource owner, on the other hand, is more interested in maximizing the utilization of the available computational resources of his site. Thus, we present two separate metrics, ProcessorUtilization (PU) and MemoryUtilization (MU), to measure the utilization of two resource components, processors and memory. The metrics are calculated the following way:

$$
M U=\frac{\sum_{i=1}^{n} M U_{i}}{\text { NodesUsed }}
$$

where:

$$
\begin{gathered}
M U_{i}=\frac{\sum_{j=1}^{k} \text { wallime }_{j} \times \text { memUsed }_{j, i}}{m p n} \\
P U=\frac{\sum_{i=1}^{n} P U_{i}}{\text { NodesUsed }}
\end{gathered}
$$

where:

$$
P U_{i}=\frac{\sum_{j=1}^{k} \text { walltime }_{j} \times n p_{j, i}}{p p n}
$$

Here, $n$ is the total number of nodes on the site, NodesUsed is the number of nodes that was actually used by any of the jobs, $k$ is the number of jobs that used a particular node, $\operatorname{memU~sed~}_{j, i}$ and $n p_{j, i}$ represent the amount of memory and the number of processors used by the job, respectively, from this node and $m p n$, ppn are the amount of memory and the number of processors on each of the nodes.

While the metrics captured by Eq. 13 and Eq. 15 are calculated by each of the individual clusters of Table 2, the utilization metrics reported in the following subsection are calculated by taking a weighted average of the utilization values. The weights are determined by the size of the cluster, i.e., the number of nodes.

\subsection{Experimental Results}

This section describes the results of the simulation using the proposed configuration selection heuristics for different workloads. The values for the above-mentioned metrics are computed for a pre-determined sample period. While defining the sample period, it was important to make sure that the simulation passed the initial transient state. Due to differences in the workload parameters and the heuristics adopted for accommodating them, it was noticed that the length of the transient period varied for different workloads. As such, different sample periods were used for different simulations while computing the metrics.

In order to determine the length of the period before the system reaches a steady state, we have used the 'randomization test' technique proposed by Yucesan [13], a test based on the commonality of batch means within the parameters of interest. As different parameters reached stability at different points of time, a conservative approach was taken in determining the overall 'startup period' by taking the longest of the transient periods.

\subsubsection{QoS optimization Results}

Figure 3 and Figure 4 show the effect of having memory usage information on optimizing the QoS objective of the job submitters. Figure 3 compares the costs of performing a single unit of computation for jobs with the Economic optimization objective. The plot clearly shows the benefit of having more memory usage information in optimizing the computation cost. As shown, for most of the size categories, costs incurred by the FullMemInfo jobs are lower than that of other types of jobs. No significant cost benefit is observed for $\mathrm{NoSeq}$ jobs despite having more information than other job categories. This could be due to such jobs being executed according to configurations with the smallest number of processors for the initial iterations in order to determine sequential memory usage. Such executions may use a larger amount of memory per processor and hence greater cost since costs are determined by the processor equivalency (discussed in Section 3.3.1).

Figure 4 compares the amount of computation completed for Urgent jobs within a given sample period. It is evident that, for most of the size categories, information on memory usage has very little impact on this metric. The primary reason behind the low impact is the way we modelled the grid resources. As shown in Table 2, in most cases, resources come with 1GB of memory per processor. Due to the urgency of completion, jobs from this class typically use a higher number of processors. For most of the parallel instances of small, medium and large jobs, the per processor memory requirement is below this amount. So, even in absence of the memory usage information, the jobs were able to run 


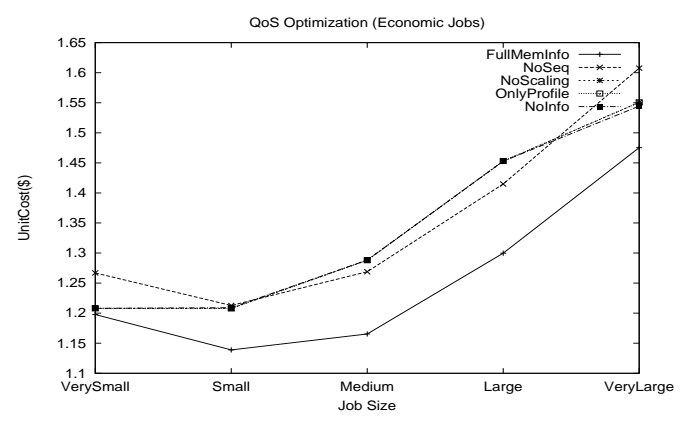

Figure 3. Optimization for Cost

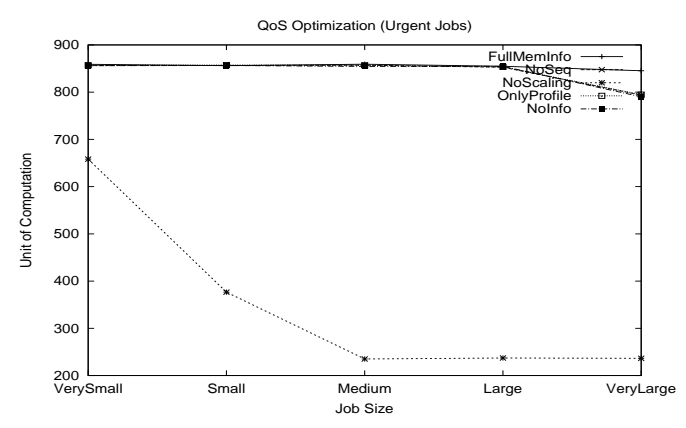

Figure 4. Optimization for Work Done

successfully by requesting the default amount, i.e., per processor memory allocation of the nodes. However, if a job's memory requirement becomes larger to the point where the per processor memory requirement is beyond the default amount, a slight advantage is noticed for FullMemInfo jobs. A deeper look into the simulation traces show that comparatively lower performance of jobs with less information can be attributed to the initial failures of these jobs due to underestimating the memory usage.

Also, according to Figure 4, we do not observe any significant performance advantage of NoSeq jobs over others with less availability of information. Due to their optimization objective, jobs tend to use similar processor counts in all their iterations. So, even in absence of sequential and scaling information on memory usage, OnlyProfile and NoInfo jobs were able to make accurate predictions in their later iterations by learning their memory usage behaviour from the initial iteration. As discussed in Section 3.3, unlike Economic jobs, configuration selection approach for NoSeq jobs does not differ between the initial and later iterations, so their processor usage behaviour is very similar to OnlyProfile and NoInfo jobs.

Another issue to notice here is the poor performance of NoScaling jobs. This is particularly due to a over conservative approach used during the configuration selection process. Jobs of this category only contain information on the sequential memory usage. To avoid job failure due to running out of memory, the serial memory usage is calculated and requested for each of the processors even in case of a parallel run. It is evident from Figure 4 that, this extreme approach of job configuration has an adverse effect on the corresponding metric. Typically it results either in a higher wait time or smaller number of available processors due to a very high memory requirement. The effect is less damaging for 'VerySmall' jobs as most of the jobs of this category request memory that is within the per processor allocation for most of the computing systems in our model.

Figure 5 shows how jobs made use of the acquired resources. For this paper, we particularly studied the usage efficiency of two resource components, CPU and memory. Also, only jobs with the Economic optimization objective were included for this study. It is clear that, jobs with more memory usage information (FullMemInfo and NoSeq jobs) are able to optimize the usage of both resource components more effectively than jobs with little or no information on memory usage. As discussed in Section 3.3, submitters of Economic jobs aim to optimize for the efficient usage of resources. However, due to difference in the information availability, for different workloads, the UserMetaScheduler have different levels of ability to optimize for these two resource components. In absence of the memory usage 


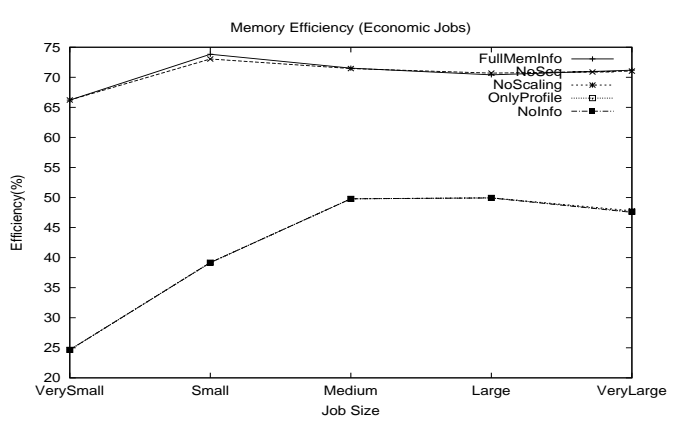

(a) Memory Efficiency

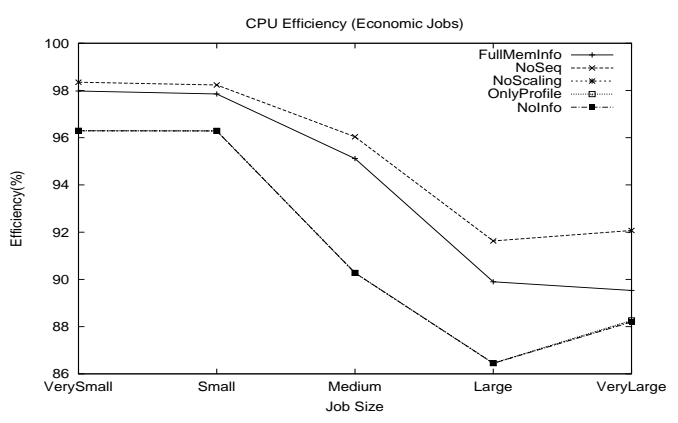

(b) CPU Efficiency

Figure 5. Resource Usage Efficiency for Economic Jobs

information, the selection of configuration is entirely based on the performance model, indicating the CPU usage efficiency of a configuration, whereas for jobs with memory information, it is possible to find a configuration that balances both the efficiency metric. It is evident from Figure 5(a) that, the selection mechanism was able to achieve a significantly higher memory efficiency for these jobs. As for NoScaling, OnlyProfile and NoInfo jobs, the selection process only considered the CPU efficiency. Although a higher efficiency values were expected for these jobs, as Figure 5(b) shows, this was not the case. It is specifically because of execution failures of jobs due to underestimating the memory usage.

Another point to notice here is the relatively higher CPU efficiency exhibited by the NoSeq jobs compare to FullMemInfo jobs. Without a knowledge about the memory usage for the initial iteration, jobs are executed on a small number of processors which results in a higher CPU usage efficiency than FullMemInfo jobs that attempt to balance the usage efficiency of two resource components. The effect of pre-mature job termination is smaller of a factor than other jobs as it can only happen during the very first iteration.

\subsubsection{Resource Utilization Results}

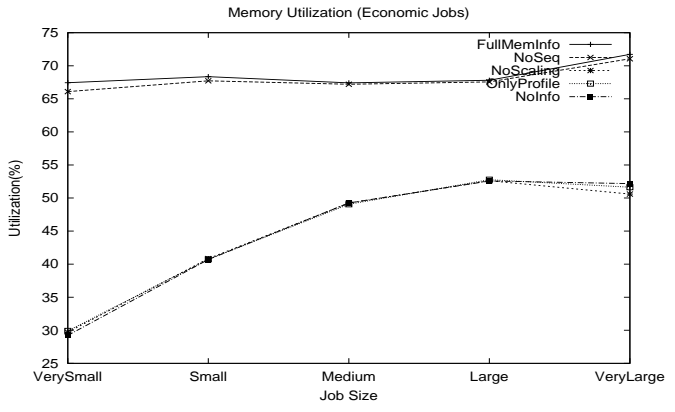

(a) Memory Utilization

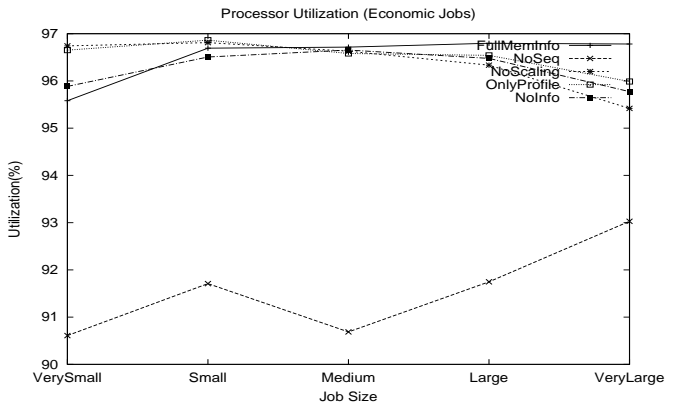

(b) Processor Utilization

Figure 6. Overall Node Utilization(Economic Jobs)

Figure 6 and Figure 7 show the cumulative resource utilization from the perspective of the resource owners. It is noticed that, the effect of memory usage information is more apparent for Economic jobs than Urgent jobs. This is due 


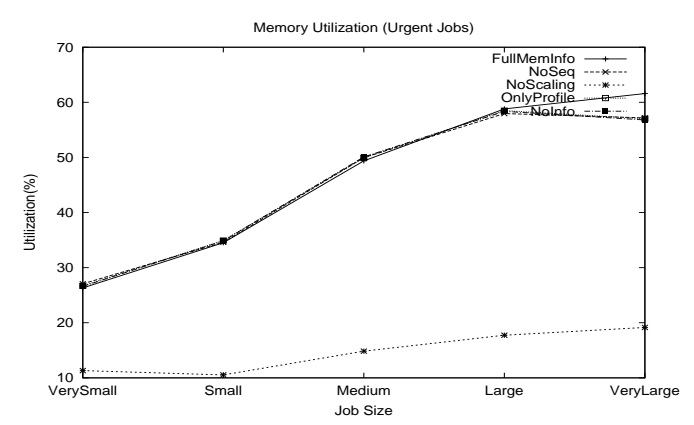

(a) Memory Utilization

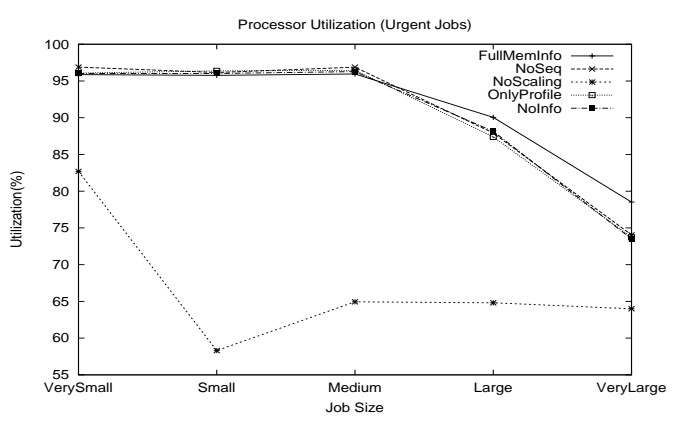

(b) Processor Utilization

\section{Figure 7. Overall Node Utilization(Urgent Jobs)}

to the difference in choosing the ranking parameter (as discussed in Section 3.3.1) during the configuration selection for these two types of jobs.

As shown in Figure 6(a), for most job sizes, a steady utilization benefit for memory is observed with jobs with more memory usage information. Essentially it is an indication to the fact that, it is easier for the resource manager to collocate jobs within a node if information about job's memory usage is known. As it is assumed that, the processors are allocated using a 'First-fit' strategy, we do not observe any significant variation in terms of the processors usage (Figure 6(b)) for most of the job types with the NoSeq jobs being the only exception. A relatively low processor utilization is due to the policy of using smaller number of processors during the initial iterations which resulted in a higher memory requirement for each of the processors used.

Figure 7(a) and Figure 7(b) show the memory and processor utilization of the Urgent jobs, respectively. Due to invariance in the number of processors used and the assumed processor allocation strategy, for most of the size categories no difference is observed in the utilization values. However, a slight advantage is noticed for both memory and processor for 'VeryLarge' jobs. This is possibly because the jobs with no information typically request more memory than FullMemInfo jobs and often for jobs of this size the requested amount exceeds the memory allocated for each processor of a node.

It is observed that, on average, the processor utilization is significantly lower for Urgent jobs compare to Economic jobs. The variation becomes more eminent as the job sizes are increased. For most of the 'Large' and 'VeryLarge' jobs, the per processor memory requirement exceeds what is allocated on most of the resources (1GB). Executing such jobs on lattice, matrix or icel_l-ice54_14 resulted in a lower processor utilization. To some extent, this was avoided in cases of Economic jobs where the preference was given to configurations that would potentially result in higher CPU efficiency. It is observed that, the heuristics resulted in a better distribution of jobs, where smaller jobs within the workload are chosen to run on systems with smaller nodes (in terms of per processor memory) and bigger jobs mostly ran on larger nodes.

We also notice poor utilization both for processors and memory in case of NoScaling jobs. The approach of over estimating the memory usage, mentioned in the discussion of Figure 7, has counterproductive implications for the overall utilization of the resources.

\section{Conclusion and Future Work}

We have proposed a framework to support automation of the processes related to execution management in grid environments. The model driven nature of the framework gives it the ability to manage execution of legacy scientific applications without the need to be customized for the framework. The framework adopts a heuristics based approach to generate configurations for jobs based on the optimization objective of the submitter, resource usage behaviour of the applications, performance, and the load of the execution platform.

A prototype implementation of the framework has been used as part of a simulation study to examine the impact different amounts of memory usage information has on achieving the job submitters QoS objectives and on the utilization of resources. Based on heuristics employed in the prototype it is evident that incorporating memory usage 
information in the configuration selection process yields a substantial advantage when a user is trying to make the most efficient use of the resources (Economic QoS objective). Memory usage information has little impact in the case where the user is trying to minimize the overall completion time of the job (Urgent QoS objective). We also observe that the overall utilization of the resource components is effected by the criterion used during the configuration selection. Use of memory usage information enables the resources to achieve a significantly higher utilization, primarily for the Economic case, even when employing a very simple scheduling and allocation strategy.

We plan to extend the current application model to include constructs that will be able to provide a more precise prediction of performance under different configurations and incorporate it into the framework. We are currently studying the ability of the framework to deal with load information with different levels of accuracy. Also, we plan to further investigate the limitations of some of the configuration selection heuristics that performed poorly in some cases such as those applied when no scaling information is present. Currently, the system uses various runtime information to adapt itself to the resource and application dynamics. However, the strategies used by the system are static. Work will be carried out to include a feedback control mechanism to the framework to enable the system to be more adaptive.

\section{References}

[1] F. Berman, A. Chien, K. Cooper, J. Dongarra, I. Foster, D. Gannon, L. Johnsson, K. Kennedy, C. Kesselman, J. MellorCrumme, D. Reed, L. Torczon, and R. Wolski. The GrADS Project: Software Support for High-Level Grid Application Development. International Journal of High Performance Computing Applications, 15(4):327-344, 2001.

[2] T. Elahi, C. Kiddle, D. Kivi, N. Markatchev, and R. Simmonds. Dynamic Job Reconfiguration in Grid Computing Environments. In Proceedings of the 14th HP Software University Association (HP-SUA) Workshop, pages 17-28, 2007.

[3] T. Elahi, C. Kiddle, and R. Simmonds. Models for Grid Applications and Jobs. In HPCS '08: Proceedings of the 2008 22nd International Symposium on High Performance Computing Systems and Applications, pages 52-58, Washington, DC, USA, 2008. IEEE Computer Society.

[4] T. Elahi, C. Kiddle, and R. Simmonds. Modelling Memory Requirements for Grid Applications. In IPDPS '09: Proceedings of the 2009 IEEE International Symposium on Parallel\&Distributed Processing, pages 1-8, Washington, DC, USA, 2009. IEEE Computer Society.

[5] F. Gomes, S. Franks, B. Unger, Z. Xiao, J. Cleary, and A. Covington. Simkit: A High Performance Logical Process Simulation Class Library in C++. In Proceedings of the 1995 Winter Simulation Conference, pages 706-713, 1995.

[6] E. Huedo, R. S. Montero, and I. M. Llorente. A Framework for Adaptive Execution in Grids. Software: Practice and Experience, 34(7):631-651, 2004.

[7] C. Kiddle and D. Kivi. GRC Model Schema 1.0.0 Specification, 2007.

[8] C. Kiddle, D. Kivi, and R. Simmonds. Model-driven Automation in Grid Environments. Proceedings of the Fourth International Symposium on Frontiers in Networking with Applications, 2008.

[9] K. Kurowski, B. Ludwiczak, J. Nabrzyski, A. Oleksiak, and J. Pukacki. Dynamic Grid Scheduling with Job Migration and Rescheduling in the Gridlab Resource Management System. Sci. Program., 12(4):263-273, 2004.

[10] S. Lacour, C. Perez, and T. Priol. Generic Application Description Model: Toward Automatic Deployment of Applications on Computational Grids. In GRID '05: Proceedings of the 6th IEEE/ACM International Workshop on Grid Computing, pages 284-287, Washington, DC, USA, 2005. IEEE Computer Society.

[11] E. Seidel, G. Allen, A. Merzky, and J. Nabrzyski. Gridlab: a Grid Application Toolkit and Testbed. Future Gener. Comput. Syst., 18(8):1143-1153, 2002.

[12] S. S. Vadhiyar and J. J. Dongarra. Self Adaptivity in Grid Computing. Concurrency and Computation: Practice and Experience, 17:235-257, 2005.

[13] E. Yucesan. Randomization Tests for Initialization Bias in Simulation Output. Naval Research Logistics, 40(5):643-663, 1993. 